\title{
Antiperiodic Boundary Value Problems for Second-Order Impulsive Ordinary Differential Equations
}

\author{
Chuanzhi Bai \\ Department of Mathematics, Huaiyin Teachers College, Huaian, Jiangsu 223300, China \\ Correspondence should be addressed to Chuanzhi Bai, czbai8@sohu.com \\ Received 23 July 2008; Accepted 23 November 2008 \\ Recommended by Raul F. Manasevich \\ We consider a second-order ordinary differential equation with antiperiodic boundary conditions \\ and impulses. By using Schaefer's fixed-point theorem, some existence results are obtained. \\ Copyright (C) 2008 Chuanzhi Bai. This is an open access article distributed under the Creative \\ Commons Attribution License, which permits unrestricted use, distribution, and reproduction in \\ any medium, provided the original work is properly cited.
}

\section{Introduction}

Impulsive differential equations, which arise in biology, physics, population dynamics, economics, and so forth, are a basic tool to study evolution processes that are subjected to abrupt in their states (see [1-4]). Many literatures have been published about existence of solutions for first-order and second-order impulsive ordinary differential equations with boundary conditions [5-19], which are important for complementing the theory of impulsive equations. In recent years, the solvability of the antiperiodic boundary value problems of first-order and second-order differential equations were studied by many authors, for example, we refer to [20-32] and the references therein. It should be noted that antiperiodic boundary value problems appear in physics in a variety of situations [33, 34]. Recently, the existence results were extended to antiperiodic boundary value problems for first-order impulsive differential equations [35, 36]. Very recently, Wang and Shen [37] investigated the antiperiodic boundary value problem for a class of second-order differential equations by using Schauder's fixed point theorem and the lower and upper solutions method.

Inspired by [35-37], in this paper, we investigate the antiperiodic boundary value problem for second-order impulsive nonlinear differential equations of the form

$$
\begin{gathered}
u^{\prime \prime}(t)+f(t, u(t))=0, \quad t \in J_{0}=J \backslash\left\{t_{1}, \ldots, t_{m}\right\}, \\
\Delta u\left(t_{k}\right)=I_{k}\left(u\left(t_{k}\right)\right), \quad k=1, \ldots, m,
\end{gathered}
$$




$$
\begin{gathered}
\Delta u^{\prime}\left(t_{k}\right)=I_{k}^{*}\left(u\left(t_{k}\right)\right), \quad k=1, \ldots, m, \\
u(0)+u(T)=0, \quad u^{\prime}(0)+u^{\prime}(T)=0,
\end{gathered}
$$

where $J=[0, T], 0<t_{1}<t_{2}<\cdots<t_{m}<T, f:[0, T] \times R \rightarrow R$ is continuous on $(t, x) \in J_{0} \times R$, $f\left(t_{k}^{+}, x\right):=\lim _{t \rightarrow t_{k}^{+}} f(t, x), f\left(t_{k}^{-}, x\right):=\lim _{t \rightarrow t_{k}^{-}} f(t, x)$ exist, $f\left(t_{k^{-}}^{-} x\right)=f\left(t_{k}, x\right) ; \Delta u\left(t_{k}\right)=u\left(t_{k}^{+}\right)-$ $u\left(t_{k}^{-}\right), \Delta u^{\prime}\left(t_{k}\right)=u^{\prime}\left(t_{k}^{+}\right)-u^{\prime}\left(t_{k}^{-}\right) ; I_{k}, I_{k}^{*} \in C(R, R)$.

To the best of the authors knowledge, no one has studied the existence of solutions for impulsive antiperiodic boundary value problem (1). The following Schaefer's fixed-point theorem is fundamental in the proof of our main results.

Lemma 1.1 (see [38] (Schaefer)). Let $E$ be a normed linear space with $H: E \rightarrow E$ a compact operator. If the set

$$
S:=\{x \in E \mid x=\lambda H x, \text { for some } \lambda \in(0,1)\}
$$

is bounded, then $H$ has at least one fixed point.

The paper is formulated as follows. In Section 2, some definitions and lemmas are given. In Section 3, we obtain two new existence theorems by using Schaefer's fixed point theorem. In Section 4, an illustrative example is given to demonstrate the effectiveness of the obtained results.

\section{Preliminaries}

In order to define the concept of solution for (1), we introduce the following spaces of functions:

$P C(J)=\left\{u: J \rightarrow R: u\right.$ is continuous for any $t \in J_{0}, u\left(t_{k}^{+}\right), u\left(t_{k}^{-}\right)$exist, and $u\left(t_{k}^{-}\right)=$ $\left.u\left(t_{k}\right), k=1, \ldots, m\right\}$,

$P C^{1}(J)=\left\{u: J \rightarrow R: u\right.$ is continuously differentiable for any $t \in J_{0}, u^{\prime}\left(t_{k}^{+}\right), u^{\prime}\left(t_{k}^{-}\right)$ exist, and $\left.u^{\prime}\left(t_{k}^{-}\right)=u^{\prime}\left(t_{k}\right), k=1, \ldots, m\right\}$.

$P C(J)$ and $P C^{1}(J)$ are Banach space with the norms

$$
\begin{gathered}
\|u\|_{P C}=\sup _{t \in J}|u(t)|, \\
\|u\|_{P C^{1}}=\max \left\{\|u\|_{P C},\left\|u^{\prime}\right\|_{P C}\right\} .
\end{gathered}
$$

A solution to the impulsive BVP (1) is a function $u \in P C^{1}(J) \cap C^{2}\left(J_{0}\right)$ that satisfies (1) for each $t \in J$.

Consider the following impulsive BVP with $\lambda>0$

$$
\begin{gathered}
-u^{\prime \prime}(t)+\lambda^{2} u(t)=\sigma(t), \quad t \in J_{0}, \\
\Delta u\left(t_{k}\right)=I_{k}\left(u\left(t_{k}\right)\right), \quad k=1, \ldots, m, \\
\Delta u^{\prime}\left(t_{k}\right)=I_{k}^{*}\left(u\left(t_{k}\right)\right), \quad k=1, \ldots, m, \\
u(0)+u(T)=0, \quad u^{\prime}(0)+u^{\prime}(T)=0,
\end{gathered}
$$

where $\sigma \in P C(J)$. 
For convenience, we set $I_{k}=I_{k}\left(u\left(t_{k}\right)\right), I_{k}^{*}=I_{k}^{*}\left(u\left(t_{k}\right)\right)$.

Lemma 2.1. $u \in P C^{1}(J) \cap C^{2}\left(J_{0}\right)$ is a solution of (2.2) if and only if $u \in P C^{1}(J)$ is a solution of the impulsive integral equation

$$
u(t)=\int_{0}^{T} G(t, s) \sigma(s) d s+\sum_{k=1}^{m}\left[G\left(t, t_{k}\right)\left(-I_{k}^{*}\right)+W\left(t, t_{k}\right) I_{k}\right]
$$

where

$$
\begin{gathered}
G(t, s)=\frac{1}{2 \lambda} \begin{cases}\frac{e^{-\lambda(t-s)}}{1+e^{-\lambda T}}-\frac{e^{\lambda(t-s)}}{1+e^{\lambda T}}, & 0 \leq s<t \leq T, \\
\frac{e^{\lambda(T+t-s)}}{1+e^{\lambda T}}-\frac{e^{-\lambda(T+t-s)}}{1+e^{-\lambda T}}, & 0 \leq t \leq s \leq T,\end{cases} \\
W(t, s)=\frac{1}{2} \begin{cases}\frac{e^{-\lambda(t-s)}}{1+e^{-\lambda T}}+\frac{e^{\lambda(t-s)}}{1+e^{\lambda T}}, & 0 \leq s<t \leq T, \\
-\frac{e^{\lambda(T+t-s)}}{1+e^{\lambda T}}-\frac{e^{-\lambda(T+t-s)}}{1+e^{-\lambda T}}, & 0 \leq t \leq s \leq T .\end{cases}
\end{gathered}
$$

Proof. If $u \in P C^{1}(J) \cap C^{2}\left(J_{0}\right)$ is a solution of (2.2), setting

$$
v(t)=u^{\prime}(t)+\lambda u(t)
$$

then, by the first equation of (2.2) we have

$$
v^{\prime}(t)-\lambda v(t)=-\sigma(t), \quad t \neq t_{k}
$$

Multiplying (2.6) by $e^{-\lambda t}$ and integrating on $\left[0, t_{1}\right)$ and $\left(t_{1}, t\right]\left(t_{1}<t \leq t_{2}\right)$, respectively, we get

$$
\begin{gathered}
e^{-\lambda t_{1}} v\left(t_{1}^{-}\right)-v(0)=-\int_{0}^{t_{1}} \sigma(s) e^{-\lambda s} d s, \\
e^{-\lambda t} v(t)-e^{-\lambda t_{1}} v\left(t_{1}^{+}\right)=-\int_{t_{1}}^{t} \sigma(s) e^{-\lambda s} d s, \quad t_{1}<t \leq t_{2} .
\end{gathered}
$$

So

$$
v(t)=e^{\lambda t}\left[v(0)-\int_{0}^{t} e^{-\lambda s} \sigma(s) d s+e^{-\lambda t_{1}} \Delta v\left(t_{1}\right)\right], \quad t_{1}<t \leq t_{2}
$$

In the same way, we can obtain that

$$
v(t)=e^{\lambda t}\left[v(0)-\int_{0}^{t} e^{-\lambda s} \sigma(s) d s+\sum_{0<t_{k}<t} e^{-\lambda t_{k}}\left(I_{k}^{*}+\lambda I_{k}\right)\right], \quad t \in J,
$$


where $v(0)=u^{\prime}(0)+\lambda u(0)$. Integrating (2.5), we have

$$
u(t)=e^{-\lambda t}\left[u(0)+\int_{0}^{t} v(s) e^{\lambda s} d s+\sum_{0<t_{k}<t} e^{\lambda t_{k}} I_{k}\right], \quad t \in J .
$$

By (2.9), we get

$$
\begin{gathered}
\int_{0}^{t} v(s) e^{\lambda s} d s=\frac{1}{2 \lambda}\left[v(0)\left(e^{2 \lambda t}-1\right)-\int_{0}^{t}\left(e^{2 \lambda t}-e^{2 \lambda s}\right) \sigma(s) e^{-\lambda s} d s\right. \\
\left.+\sum_{0<t_{k}<t}\left(e^{2 \lambda t}-e^{2 \lambda t_{k}}\right) e^{-\lambda t_{k}}\left(I_{k}^{*}+\lambda I_{k}\right)\right] .
\end{gathered}
$$

Substituting (2.11) into (2.10), we obtain

$$
\begin{aligned}
& u(t)=\frac{1}{2 \lambda}\left[\left(\lambda u(0)-u^{\prime}(0)\right) e^{-\lambda t}+\left(u^{\prime}(0)+\lambda u(0)\right) e^{\lambda t}\right. \\
& +\int_{0}^{t}\left(e^{-\lambda(t-s)}-e^{\lambda(t-s)}\right) \sigma(s) d s+\sum_{0<t_{k}<t} e^{\lambda\left(t-t_{k}\right)}\left(I_{k}^{*}+\lambda I_{k}\right) \\
& \left.-\sum_{0<t_{k}<t} e^{-\lambda\left(t-t_{k}\right)}\left(I_{k}^{*}-\lambda I_{k}\right)\right], \quad t \in J, \\
& u^{\prime}(t)=\frac{1}{2}\left[-\left(\lambda u(0)-u^{\prime}(0)\right) e^{-\lambda t}+\left(u^{\prime}(0)+\lambda u(0)\right) e^{\lambda t}\right. \\
& -\int_{0}^{t}\left(e^{-\lambda(t-s)}+e^{\lambda(t-s)}\right) \sigma(s) d s+\sum_{0<t_{k}<t} e^{\lambda\left(t-t_{k}\right)}\left(I_{k}^{*}+\lambda I_{k}\right) \\
& \left.+\sum_{0<t_{k}<t} e^{-\lambda\left(t-t_{k}\right)}\left(I_{k}^{*}-\lambda I_{k}\right)\right], \quad t \in J .
\end{aligned}
$$

In view of $u(0)+u(T)=0$ and $u^{\prime}(0)+u^{\prime}(T)=0$, we have

$$
\begin{gathered}
u^{\prime}(0)+\lambda u(0)=\frac{1}{1+e^{\lambda T}}\left[\int_{0}^{T} e^{\lambda(T-s)} \sigma(s) d s-\sum_{0<t_{k}<T} e^{\lambda\left(T-t_{k}\right)}\left(I_{k}^{*}+\lambda I_{k}\right)\right], \\
\lambda u(0)-u^{\prime}(0)=\frac{1}{1+e^{-\lambda T}}\left[-\int_{0}^{T} e^{-\lambda(T-s)} \sigma(s) d s+\sum_{0<t_{k}<T} e^{-\lambda\left(T-t_{k}\right)}\left(I_{k}^{*}-\lambda I_{k}\right)\right] .
\end{gathered}
$$

Substituting (2.14) into (2.12), by routine calculation, we can get (2.3).

Conversely, if $u$ is a solution of (2.3), then direct differentiation of (2.3) gives $-u^{\prime \prime}(t)=$ $\sigma(t)-\lambda^{2} u(t), t \neq t_{k}$. Moreover, we obtain $\left.\Delta u\right|_{t=t_{k}}=I_{k}\left(u\left(t_{k}\right)\right),\left.\Delta u^{\prime}\right|_{t=t_{k}}=I_{k}^{*}\left(u\left(t_{k}\right)\right), u(0)+u(T)=0$ and $u^{\prime}(0)+u^{\prime}(T)=0$. Hence, $u \in P C^{1}(J) \cap C^{2}\left(J_{0}\right)$ is a solution of (2.2). 
Remark 2.2. We call $G(t, s)$ above the Green function for the following homogeneous BVP:

$$
\begin{gathered}
-u^{\prime \prime}(t)+\lambda^{2} u(t)=0, \quad t \in J, \\
u(0)+u(T)=0, \quad u^{\prime}(0)+u^{\prime}(T)=0 .
\end{gathered}
$$

Define a mapping $A: P C^{1}(J) \rightarrow P C^{1}(J)$ by

$$
A u(t)=\int_{0}^{T} G(t, s)\left[f(s, u(s))+\lambda^{2} u(s)\right] d s+\sum_{k=1}^{m}\left[G\left(t, t_{k}\right)\left(-I_{k}^{*}\right)+W\left(t, t_{k}\right) I_{k}\right], \quad t \in[0, T] .
$$

In view of Lemma 2.1, we easily see that $u$ is a fixed point of operator $A$ if and only if $u$ is a solution to the impulsive boundary value problem (1).

It is easy to check that

$$
|G(t, s)| \leq \frac{e^{\lambda T}-1}{2 \lambda\left(1+e^{\lambda T}\right)}, \quad|W(t, s)| \leq \frac{1}{2}
$$

Lemma 2.3. If $u \in P C^{1}(J)$ and $u(0)+u(T)=0$, then

$$
\|u\|_{P C} \leq \frac{1}{2}\left(\int_{0}^{T}\left|u^{\prime}(s)\right| d s+\sum_{k=1}^{m}\left|\Delta u\left(t_{k}\right)\right|\right) .
$$

Proof. Since $u \in P C^{1}(J)$, we have

$$
u(t)=u(0)+\sum_{0<t_{k}<t} \Delta u\left(t_{k}\right)+\int_{0}^{t} u^{\prime}(s) d s .
$$

Set $t=T$, we obtain from $u(0)+u(T)=0$ that

$$
u(0)=-\frac{1}{2}\left(\sum_{k=1}^{m} \Delta u\left(t_{k}\right)+\int_{0}^{T} u^{\prime}(s) d s\right) .
$$

Substituting (2.20) into (2.19), we get

$$
\begin{aligned}
|u(t)| & =\left|\frac{1}{2}\left(\int_{0}^{t} u^{\prime}(s) d s-\int_{t}^{T} u^{\prime}(s) d s\right)+\frac{1}{2}\left(\sum_{0<t_{k}<t} \Delta u\left(t_{k}\right)-\sum_{t \leq t_{k}} \Delta u\left(t_{k}\right)\right)\right| \\
& \leq \frac{1}{2}\left(\int_{0}^{t}\left|u^{\prime}(s)\right| d s+\int_{t}^{T}\left|u^{\prime}(s)\right| d s\right)+\frac{1}{2}\left(\sum_{0<t_{k}<t}\left|\Delta u\left(t_{k}\right)\right|+\sum_{t \leq t_{k}}\left|\Delta u\left(t_{k}\right)\right|\right) \\
& =\frac{1}{2}\left(\int_{0}^{T}\left|u^{\prime}(s)\right| d s+\sum_{k=1}^{m}\left|\Delta u\left(t_{k}\right)\right|\right) .
\end{aligned}
$$

The proof is complete. 


\section{Main results}

In this section, we study the existence of solutions for BVP (1). For this purpose we assume that there exist constants $0<\eta<1$, functions $a, b, h \in C(J,[0,+\infty))$, and nonnegative constants $\alpha_{k}, \beta_{k}, \gamma_{k}, \delta_{k}(k=1,2, \ldots, m)$ such that

$$
\begin{aligned}
& \left(\mathrm{H}_{1}\right)|f(t, u)| \leq a(t)|u|+b(t)|u|^{\eta}+h(t), \text { and } \\
& \left(\mathrm{H}_{2}\right)\left|I_{k}(u)\right| \leq \alpha_{k}|u|+\beta_{k},\left|I_{k}^{*}(u)\right| \leq \gamma_{k}|u|+\delta_{k}, k=1, \ldots, m
\end{aligned}
$$

hold.

Remark 3.1. $\left(\mathrm{H}_{1}\right)$ means that the nonlinearity growths at most linearly in $u,\left(\mathrm{H}_{2}\right)$ implies that the impulses are (at most) linear.

For convenience, let

$$
\begin{gathered}
p_{1}=\frac{3}{2}\left(\int_{0}^{T} a(t) d t+\lambda^{2} T+\sum_{i=1}^{m} \gamma_{i}\right), \\
p_{2}=\frac{3}{2} \int_{0}^{T} b(t) d t \\
p_{3}=\frac{3}{2}\left(\int_{0}^{T} h(t) d t+\sum_{i=1}^{m} \delta_{i}\right) .
\end{gathered}
$$

Theorem 3.2. Suppose that conditions $\left(H_{1}\right)$ and $\left(H_{2}\right)$ are satisfied. Further assume that

$$
\frac{T}{4} q_{1}+\frac{\sqrt{T q_{1}}}{2} \sum_{i=1}^{m} \alpha_{i}+\frac{m}{4} \sum_{i=1}^{m} \alpha_{i}^{2}<1
$$

holds, where $q_{1}=\int_{0}^{T} a(t) d t+\sum_{i=1}^{m}\left(p_{1} \alpha_{i}+\gamma_{i}\right)$ and $p_{1}$ as in (3.1). Then, BVP (1) has at least one solution.

Proof. It is easy to check by Arzela-Ascoli theorem that the operator $A$ is completely continuous. Assume that $u$ is a solution of the equation

$$
u=\mu A u, \quad \mu \in(0,1) .
$$

Then,

$$
\begin{aligned}
u^{\prime \prime}(t) & =\mu(A u)^{\prime \prime}(t)=\mu\left[-f(t, u(t))-\lambda^{2} u(t)+\lambda^{2}(A u)(t)\right] \\
& =-\mu f(t, u(t))-\lambda^{2}(\mu-1) u(t), \\
-u(t) u^{\prime \prime}(t) & =\mu u(t) f(t, u(t))+\lambda^{2}(\mu-1) u^{2}(t) \leq \mu u(t) f(t, u(t)) .
\end{aligned}
$$


Integrating (3.4) from 0 to $T$, we get that

$$
u^{\prime}(T)-u^{\prime}(0)=\int_{0}^{T} u^{\prime \prime}(t) d t+\sum_{i=1}^{m} I_{i}^{*}=-\mu \int_{0}^{T} f(t, u(t)) d t-\lambda^{2}(\mu-1) \int_{0}^{T} u(t) d t+\sum_{i=1}^{m} I_{i}^{*}
$$

In view of $u^{\prime}(0)+u^{\prime}(T)=0$, we obtain by (3.6) that

$$
\left|u^{\prime}(0)\right| \leq \frac{1}{2} \int_{0}^{T}|f(t, u(t))| d t+\frac{\lambda^{2}}{2} \int_{0}^{T}|u(t)| d t+\frac{1}{2} \sum_{i=1}^{m}\left|I_{i}^{*}\right|
$$

Integrating (3.4) from 0 to $t$, we obtain that

$$
u^{\prime}(t)-u^{\prime}(0)=\int_{0}^{t} u^{\prime \prime}(s) d s+\sum_{0<t_{i}<t} I_{i}^{*}=-\mu \int_{0}^{t} f(s, u(s)) d s-\lambda^{2}(\mu-1) \int_{0}^{t} u(s) d s+\sum_{0<t_{i}<t} I_{i}^{*} .
$$

From (3.7) and (3.8), we have

$$
\begin{aligned}
\left|u^{\prime}(t)\right| & \leq\left|u^{\prime}(0)\right|+\int_{0}^{T}|f(s, u(s))| d s+\lambda^{2} \int_{0}^{T}|u(s)| d s+\sum_{i=1}^{m}\left|I_{i}^{*}\right| \\
& \leq \frac{3}{2} \int_{0}^{T}\left(a(t)|u(t)|+b(t)|u(t)|^{\eta}+h(t)\right) d t+\frac{3}{2} \lambda^{2} \int_{0}^{T}|u(t)| d t+\frac{3}{2} \sum_{i=1}^{m}\left(\gamma_{i}\|u\|_{P C}+\delta_{i}\right) \\
& \leq \frac{3}{2}\left(\|u\|_{P C} \int_{0}^{T} a(t) d t+\|u\|_{P C}^{\eta} \int_{0}^{T} b(t) d t+\int_{0}^{T} h(t) d t\right)+\frac{3}{2} \lambda^{2} T\|u\|_{P C}+\frac{3}{2} \sum_{i=1}^{m}\left(\gamma_{i}\|u\|_{P C}+\delta_{i}\right),
\end{aligned}
$$

that is,

$$
\left\|u^{\prime}\right\|_{P C} \leq \frac{3}{2}\left(\int_{0}^{T} a(t) d t+\lambda^{2} T+\sum_{i=1}^{m} \gamma_{i}\right)\|u\|_{P C}+\frac{3}{2} \int_{0}^{T} b(t) d t\|u\|_{P C}^{\eta}+\frac{3}{2} \int_{0}^{T} h(t) d t+\frac{3}{2} \sum_{i=1}^{m} \delta_{i} .
$$

Thus,

$$
\left\|u^{\prime}\right\|_{P C} \leq p_{1}\|u\|_{P C}+p_{2}\|u\|_{P C}^{\eta}+p_{3}
$$

where $p_{1}, p_{2}, p_{3}$ are as in (3.1). Integrating (3.5) from 0 to $T$, we get that

$$
-\int_{0}^{T} u(t) u^{\prime \prime}(t) d t \leq \mu \int_{0}^{T} u(t) f(t, u(t)) d t
$$


In view of $u(0)+u(T)=0$ and $u^{\prime}(0)+u^{\prime}(T)=0$, we have

$$
\begin{aligned}
\int_{0}^{T} u(t) u^{\prime \prime}(t) d t= & \int_{0}^{T} u(t) d\left(u^{\prime}(t)\right) \\
= & \int_{0}^{t_{1}} u(t) d\left(u^{\prime}(t)\right)+\int_{t_{1}}^{t_{2}} u(t) d\left(u^{\prime}(t)\right)+\cdots+\int_{t_{n}}^{T} u(t) d\left(u^{\prime}(t)\right) \\
= & \left.(t) u^{\prime}(t)\right|_{0} ^{t_{1}}-\int_{0}^{t_{1}}\left(u^{\prime}(t)\right)^{2} d t+\left.u(t) u^{\prime}(t)\right|_{t_{1}} ^{t_{2}}-\int_{t_{1}}^{t_{2}}\left(u^{\prime}(t)\right)^{2} d t \\
& +\cdots+\left.u(t) u^{\prime}(t)\right|_{t_{n}} ^{T}-\int_{t_{n}}^{T}\left(u^{\prime}(t)\right)^{2} d t \\
= & u\left(t_{1}-0\right) u^{\prime}\left(t_{1}-0\right)-u(0) u^{\prime}(0)+u\left(t_{2}-0\right) u^{\prime}\left(t_{2}-0\right)-u\left(t_{1}+0\right) u^{\prime}\left(t_{1}+0\right) \\
& +\cdots+u(T) u^{\prime}(T)-u\left(t_{n}+0\right) u^{\prime}\left(t_{n}+0\right)-\int_{0}^{T}\left(u^{\prime}(t)\right)^{2} d t \\
= & u\left(t_{1}-0\right) u^{\prime}\left(t_{1}-0\right)-u\left(t_{1}+0\right) u^{\prime}\left(t_{1}+0\right) \\
& +\cdots+u\left(t_{n}-0\right) u^{\prime}\left(t_{n}-0\right)-u\left(t_{n}+0\right) u^{\prime}\left(t_{n}+0\right)-\int_{0}^{T}\left(u^{\prime}(t)\right)^{2} d t \\
= & u\left(t_{1}-0\right) u^{\prime}\left(t_{1}-0\right)-u\left(t_{1}-0\right) u^{\prime}\left(t_{1}+0\right)+u\left(t_{1}-0\right) u^{\prime}\left(t_{1}+0\right) \\
& -u\left(t_{1}+0\right) u^{\prime}\left(t_{1}+0\right)+\cdots+u\left(t_{n}-0\right) u^{\prime}\left(t_{n}-0\right)-u\left(t_{n}-0\right) u^{\prime}\left(t_{n}+0\right) \\
& +u\left(t_{n}-0\right) u^{\prime}\left(t_{n}+0\right)-u\left(t_{n}+0\right) u^{\prime}\left(t_{n}+0\right)-\int_{0}^{T}\left(u^{\prime}(t)\right)^{2} d t \\
= & -u\left(t_{1}-0\right) I_{1}^{*}-u^{\prime}\left(t_{1}+0\right) I_{1}-\cdots-u\left(t_{n}-0\right) I_{n}^{*}-u^{\prime}\left(t_{n}+0\right) I_{n}-\int_{0}^{T}\left(u^{\prime}(t)\right)^{2} d t .
\end{aligned}
$$

Substituting (3.13) into (3.12), we obtain by $\left(\mathrm{H}_{2}\right),\left(\mathrm{H}_{3}\right)$, and (3.11) that

$$
\begin{aligned}
\int_{0}^{T}\left(u^{\prime}(t)\right)^{2} d t \leq & \mu \int_{0}^{T} u(t) f(t, u(t)) d t-u\left(t_{1}-0\right) I_{1}^{*} \\
& -u^{\prime}\left(t_{1}+0\right) I_{1}-\cdots-u\left(t_{n}-0\right) I_{n}^{*}-u^{\prime}\left(t_{n}+0\right) I_{n} \\
\leq & \mu \int_{0}^{T} u(t) f(t, u(t)) d t+\|u\|_{P C} \sum_{i=1}^{m}\left|I_{i}^{*}\right|+\left\|u^{\prime}\right\|_{P C} \sum_{i=1}^{m}\left|I_{i}\right| \\
\leq & \int_{0}^{T}\left(a(t) u^{2}(t)+b(t)|u(t)|^{1+\eta}+h(t)|u(t)|\right) d t \\
& +\|u\|_{P C} \sum_{i=1}^{m}\left(\gamma_{i}\|u\|_{P C}+\delta_{i}\right)+\left\|u^{\prime}\right\|_{P C} \sum_{i=1}^{m}\left(\alpha_{i}\|u\|_{P C}+\beta_{i}\right) \\
\leq & \|u\|_{P C}^{2} \int_{0}^{T} a(t) d t+\|u\|_{P C}^{1+\eta} \int_{0}^{T} b(t) d t+\|u\|_{P C} \int_{0}^{T} h(t) d t \\
& +\sum_{i=1}^{m} \gamma_{i}\|u\|_{P C}^{2}+\sum_{i=1}^{m} \delta_{i}\|u\|_{P C}+\left(p_{1}\|u\|_{P C}+p_{2}\|u\|_{P C}^{\eta}+p_{3}\right) \sum_{i=1}^{m}\left(\alpha_{i}\|u\|_{P C}+\beta_{i}\right) .
\end{aligned}
$$


Thus,

$$
\int_{0}^{T}\left(u^{\prime}(t)\right)^{2} d t \leq q_{1}\|u\|_{P C}^{2}+q_{2}\|u\|_{P C}^{1+\eta}+q_{3}\|u\|_{P C}+q_{4}\|u\|_{P C}^{\eta}+q_{5}
$$

where

$$
\begin{aligned}
& q_{1}=\int_{0}^{T} a(t) d t+\sum_{i=1}^{m}\left(p_{1} \alpha_{i}+\gamma_{i}\right), \\
& q_{2}=\int_{0}^{T} b(t) d t+p_{2} \sum_{i=1}^{m} \alpha_{i}, \\
& q_{3}=\int_{0}^{T} h(t) d t+\sum_{i=1}^{m}\left(p_{1} \beta_{i}+p_{3} \alpha_{i}+\delta_{i}\right), \\
& q_{4}=p_{2} \sum_{i=1}^{m} \beta_{i}, \\
& q_{5}=p_{3} \sum_{i=1}^{m} \beta_{i} .
\end{aligned}
$$

By Lemma 2.3 and (3.15), we have

$$
\begin{aligned}
\|u\|_{P C}^{2} \leq & \frac{1}{4}\left(\int_{0}^{T}\left|u^{\prime}(t)\right| d t\right)^{2}+\frac{1}{2} \int_{0}^{T}\left|u^{\prime}(t)\right| d t \sum_{i=1}^{m}\left|I_{i}\right|+\frac{1}{4}\left(\sum_{i=1}^{m}\left|I_{i}\right|\right)^{2} \\
\leq & \frac{T}{4} \int_{0}^{T}\left(u^{\prime}(t)\right)^{2} d t+\frac{\sqrt{T}}{2}\left(\int_{0}^{T}\left(u^{\prime}(t)\right)^{2} d t\right)^{1 / 2} \sum_{i=1}^{m}\left|I_{i}\right|+\frac{m}{4} \sum_{i=1}^{m}\left|I_{i}\right|^{2} \\
\leq & \frac{T}{4}\left[q_{1}\|u\|_{P C}^{2}+q_{2}\|u\|_{P C}^{1+\eta}+q_{3}\|u\|_{P C}+q_{4}\|u\|_{P C}^{\eta}+q_{5}\right] \\
& +\frac{\sqrt{T}}{2}\left[q_{1}\|u\|_{P C}^{2}+q_{2}\|u\|_{P C}^{1+\eta}+q_{3}\|u\|_{P C}+q_{4}\|u\|_{P C}^{\eta}+q_{5}\right]^{1 / 2} \\
& \times \sum_{i=1}^{m}\left(\alpha_{i}\|u\|_{P C}+\beta_{i}\right)+\frac{m}{4} \sum_{i=1}^{m}\left(\alpha_{i}^{2}\|u\|_{P C}^{2}+2 \alpha_{i} \beta_{i}\|u\|_{P C}+\beta_{i}^{2}\right) \\
= & \left(\frac{T q_{1}}{4}+\frac{\sqrt{T q_{1}}}{2} \sum_{i=1}^{m} \alpha_{i}+\frac{m}{4} \sum_{i=1}^{m} \alpha_{i}^{2}\right)\|u\|_{P C}^{2}+\cdots
\end{aligned}
$$

It follows from the above inequality and (3.2) that there exists $M_{1}>0$ such that $\|u\|_{P C} \leq M_{1}$. Hence, we get by (3.11) that

$$
\left\|u^{\prime}\right\|_{P C} \leq a_{1} M_{1}+a_{2} M_{1}^{\eta}+a_{3}:=M_{2} .
$$


Thus, $\|u\|_{P C^{1}} \leq \max \left\{M_{1}, M_{2}\right\}$. It follows from Lemma 1.1 that BVP (1) has at least one solution. The proof is complete.

Theorem 3.3. Assume that $\left(\mathrm{H}_{2}\right)$ holds. Suppose that there exist a continuous and nondecreasing function $\psi:[0, \infty) \rightarrow[0, \infty)$ and a nonnegative function $c \in C(J)$ with

$$
\left|f(t, u)+\lambda^{2} u\right| \leq c(t) \psi(|u|), \quad t \in J, u \in R .
$$

Moreover suppose that

$$
\lim \sup _{u \rightarrow \infty} \frac{\psi(u)}{u}<L
$$

holds, where

$$
L:=\frac{1-\left(\left(e^{\lambda T}-1\right) / 2 \lambda\left(1+e^{\lambda T}\right)\right) \sum_{i=1}^{m} \gamma_{i}-(1 / 2) \sum_{i=1}^{m} \alpha_{i}}{\left(\left(e^{\lambda T}-1\right) / 2 \lambda\left(1+e^{\lambda T}\right)\right) \int_{0}^{T} c(s) d s}>0 .
$$

Then, BVP (1) has at least one solution.

Proof. From (3.20), there exist $0<\varepsilon<L$ and $M>0$ such that

$$
\psi(v) \leq(L-\varepsilon) v, \quad v \geq M
$$

Thus, there exists $K>0$ such that

$$
\psi(v) \leq(L-\varepsilon) v+K, \quad v \geq 0 .
$$

Assume that $u$ is a solution of the equation

$$
u=\mu A u, \quad \mu \in(0,1) .
$$

Then, we have by (3.19), (2.17), and (3.23) that

$$
\begin{aligned}
|u(t)| & =\mu\left|\int_{0}^{T} G(t, s)\left(f(s, u(s))+\lambda^{2} u(s)\right) d s+\sum_{k=1}^{m}\left[G\left(t, t_{k}\right)\left(-I_{k}^{*}\right)+W\left(t, t_{k}\right) I_{k}\right]\right| \\
& \leq \frac{e^{\lambda T}-1}{2 \lambda\left(1+e^{\lambda T}\right)} \int_{0}^{T} c(s) \psi(|u|) d s+\frac{e^{\lambda T}-1}{2 \lambda\left(1+e^{\lambda T}\right)} \sum_{i=1}^{m}\left(\gamma_{i}\|u\|_{P C}+\delta_{i}\right)+\frac{1}{2} \sum_{i=1}^{m}\left(\alpha_{i}\|u\|_{P C}+\beta_{i}\right) .
\end{aligned}
$$


Thus, we have

$$
\begin{aligned}
\|u\|_{P C} \leq & \frac{e^{\lambda T}-1}{2 \lambda\left(1+e^{\lambda T}\right)} \int_{0}^{T} c(s) d s\left((L-\varepsilon)\|u\|_{P C}+K\right) \\
& +\left(\frac{e^{\lambda T}-1}{2 \lambda\left(1+e^{\lambda T}\right)} \sum_{i=1}^{m} \gamma_{i}+\frac{1}{2} \sum_{i=1}^{m} \alpha_{i}\right)\|u\|_{P C}+\frac{e^{\lambda T}-1}{2 \lambda\left(1+e^{\lambda T}\right)} \sum_{i=1}^{m} \delta_{i}+\frac{1}{2} \sum_{i=1}^{m} \beta_{i}
\end{aligned}
$$

that is,

$$
\varepsilon \frac{e^{\lambda T}-1}{2 \lambda\left(1+e^{\lambda T}\right)} \int_{0}^{T} c(s) d s\|u\|_{P C} \leq \frac{e^{\lambda T}-1}{2 \lambda\left(1+e^{\lambda T}\right)} K \int_{0}^{T} c(s) d s+\frac{e^{\lambda T}-1}{2 \lambda\left(1+e^{\lambda T}\right)} \sum_{i=1}^{m} \delta_{i}+\frac{1}{2} \sum_{i=1}^{m} \beta_{i},
$$

which implies that there exists $M_{3}>0$ such that $\|u\|_{P C} \leq M_{3}$. By (3.7), (3.8), and (3.23), we get

$$
\begin{aligned}
\left|u^{\prime}(t)\right| & \leq \frac{3}{2} \int_{0}^{T}|f(s, u(s))| d s+\frac{3}{2} \lambda^{2} \int_{0}^{T}|u(s)| d s+\frac{3}{2} \sum_{i=1}^{m}\left|I_{i}^{*}\right| \\
& \leq \frac{3}{2} \int_{0}^{T}\left|f(s, u(s))+\lambda^{2} u(s)\right| d s+3 \lambda^{2} \int_{0}^{T}|u(s)| d s+\frac{3}{2} \sum_{i=1}^{m}\left|I_{i}^{*}\right| \\
& \leq \frac{3}{2} \int_{0}^{T} c(s) \psi(|u(s)|) d s+3 \lambda^{2} \int_{0}^{T}|u(s)| d s+\frac{3}{2} \sum_{i=1}^{m}\left(\gamma_{i}\|u\|_{P C}+\delta_{i}\right) \\
& \leq \frac{3}{2} \int_{0}^{T} c(s) d s\left(L\|u\|_{P C}+K\right)+3 \lambda^{2} T\|u\|_{P C}+\frac{3}{2} \sum_{i=1}^{m}\left(\gamma_{i}\|u\|_{P C}+\delta_{i}\right),
\end{aligned}
$$

which implies that

$$
\begin{aligned}
\left\|u^{\prime}\right\|_{P C} & \leq\left(\frac{3 L}{2} \int_{0}^{T} c(s) d s+3 \lambda^{2} T+\frac{3}{2} \sum_{i=1}^{m} \gamma_{i}\right)\|u\|_{P C}+\frac{3 K}{2} \int_{0}^{T} c(s) d s+\frac{3}{2} \sum_{i=1}^{m} \delta_{i} \\
& \leq \frac{3}{2}\left(L \int_{0}^{T} c(s) d s+2 \lambda^{2} T+\sum_{i=1}^{m} \gamma_{i}\right) M_{3}+\frac{3 K}{2} \int_{0}^{T} c(s) d s+\frac{3}{2} \sum_{i=1}^{m} \delta_{i}:=M_{4} .
\end{aligned}
$$

Hence, $\|u\|_{P C^{1}} \leq \max \left\{M_{3}, M_{4}\right\}$. It follows from Lemma 1.1 that BVP (1) has at least one solution. The proof is complete.

\section{Example}

In this section, we give an example to illustrate the effectiveness of our results. 
Example 4.1. Consider the problem

$$
\begin{gathered}
u^{\prime \prime}(t)+\frac{1}{\pi} u(t) \cos ^{2} t+\frac{1}{2} e^{t} u^{1 / 2}(t)+1+\tan t=0, \quad t \in\left[0, \frac{\pi}{2}\right] \backslash\left\{\frac{\pi}{3}\right\}, \\
\Delta u\left(t_{1}\right)=\frac{1}{3} \sin \left(u\left(t_{1}\right)\right)+\frac{1}{4}, \quad \Delta u^{\prime}\left(t_{1}\right)=\frac{1}{2} u\left(t_{1}\right)+\frac{1}{3}, \quad t_{1}=\frac{\pi}{3}, \\
u(0)+u(T)=0, \quad u^{\prime}(0)+u^{\prime}(T)=0,
\end{gathered}
$$

Let $f(t, u)=(1 / \pi) u \cos ^{2} t+(1 / 2) e^{t} u^{1 / 2}+1+\tan t, I_{1}(u)=(1 / 3) \sin u+(1 / 4), I_{1}^{*}(u)=(1 / 2) u+$ $(1 / 3), T=(\pi / 2), J=[0, \pi / 2]$. It is easy to show that

$$
|f(t, u)| \leq a(t)|u|+b(t)|u|^{1 / 2}+h(t),
$$

where $a(t)=(1 / \pi) \cos ^{2} t, b(t)=(1 / 2) e^{t}, h(t)=1+\tan t$. And

$$
\left|I_{1}(u)\right| \leq \frac{1}{3}|u|+\frac{1}{4}, \quad\left|I_{1}^{*}(u)\right| \leq \frac{1}{2}|u|+\frac{1}{3} .
$$

Thus, $\left(\mathrm{H}_{1}\right)$ and $\left(\mathrm{H}_{2}\right)$ hold. Obviously, $\alpha_{1}=1 / 3, \beta_{1}=1 / 4, \gamma_{1}=1 / 2, \delta_{1}=1 / 3$, and $m=1$. Let $\lambda^{2}=1 / 4 \pi$, we have

$$
\begin{aligned}
& p_{1}=\frac{3}{2}\left(\int_{0}^{T} a(t) d t+2 \lambda^{2} T+\sum_{i=1}^{m} \gamma_{i}\right)=\frac{3}{2}\left(\frac{1}{4}+\frac{1}{4}+\frac{1}{2}\right)=\frac{3}{2} \\
& q_{1}=\int_{0}^{T} a(t) d t+\sum_{i=1}^{m}\left(p_{1} \alpha_{i}+\gamma_{i}\right)=\frac{1}{4}+\frac{3}{2} \cdot \frac{1}{3}+\frac{1}{2}=\frac{5}{4} .
\end{aligned}
$$

Therefore,

$$
\frac{T q_{1}}{4}+\frac{\sqrt{T q_{1}}}{2} \sum_{i=1}^{m} \alpha_{i}+\frac{m}{4} \sum_{i=1}^{m} \alpha_{i}^{2}=0.7522<1
$$

which implies that (3.2) holds. So, all the conditions of Theorem 3.2 are satisfied. By Theorem 3.2, antiperiod boundary value problem (4.1) has at least one solution.

\section{Acknowledgments}

The author would like to thank the referees for their valuable suggestions and comments. This project is supported by the National Natural Science Foundation of China (10771212) and the Natural Science Foundation of Jiangsu Education Office (06KJB110010).

\section{References}

[1] V. Lakshmikantham, D. D. Baĭnov, and P. S. Simeonov, Theory of Impulsive Differential Equations, vol. 6 of Series in Modern Applied Mathematics, World Scientific, Singapore, 1989.

[2] A. M. Samoulenko and N. A. Perestyuk, Impulsive Differential Equations, vol. 14 of World Scientific Series on Nonlinear Science. Series A: Monographs and Treatises, World Scientific, Singapore, 1995. 
[3] S. T. Zavalishchin and A. N. Sesekin, Dynamic Impulse Systems: Theory and Application, vol. 394 of Mathematics and Its Applications, Kluwer Academic Publishers, Dordrecht, The Netherlands, 1997.

[4] X. Liu, Ed., "Advances in impulsive differential equations," Dynamics of Continuous, Discrete $\mathcal{E}$ Impulsive Systems. Series A, vol. 9, no. 3, pp. 313-462, 2002.

[5] C. Bai and D. Yang, "Existence of solutions for second-order nonlinear impulsive differential equations with periodic boundary value conditions," Boundary Value Problems, vol. 2007, Article ID 41589, 13 pages, 2007.

[6] J. Li and J. Shen, "Periodic boundary value problems for second order differential equations with impulses," Nonlinear Studies, vol. 12, no. 4, pp. 391-400, 2005.

[7] J. J. Nieto, "Periodic boundary value problems for first-order impulsive ordinary differential equations," Nonlinear Analysis: Theory, Methods E Applications, vol. 51, no. 7, pp. 1223-1232, 2002.

[8] J. Chen, C. C. Tisdell, and R. Yuan, "On the solvability of periodic boundary value problems with impulse," Journal of Mathematical Analysis and Applications, vol. 331, no. 2, pp. 902-912, 2007.

[9] M. Benchohra, J. Henderson, and S. Ntouyas, Impulsive Differential Equations and Inclusions, vol. 2 of Contemporary Mathematics and Its Applications, Hindawi, New York, NY, USA, 2006.

[10] T. Jankowski and J. J. Nieto, "Boundary value problems for first-order impulsive ordinary differential equations with delay arguments," Indian Journal of Pure and Applied Mathematics, vol. 38, no. 3, pp. 203-211, 2007.

[11] G. Zeng, F. Wang, and J. J. Nieto, "Complexity of a delayed predator-prey model with impulsive harvest and Holling type II functional response," Advances in Complex Systems, vol. 11, no. 1, pp. 7797, 2008.

[12] M. U. Akhmetov, A. Zafer, and R. D. Sejilova, "The control of boundary value problems for quasilinear impulsive integro-differential equations," Nonlinear Analysis: Theory, Methods \& Applications, vol. 48, no. 2, pp. 271-286, 2002.

[13] J. J. Nieto and D. O'Regan, "Variational approach to impulsive differential equations," Nonlinear Analysis: Real World Applications, vol. 10, no. 2, pp. 680-690, 2009.

[14] H. Zhang, L. Chen, and J. J. Nieto, "A delayed epidemic model with stage-structure and pulses for pest management strategy," Nonlinear Analysis: Real World Applications, vol. 9, no. 4, pp. 1714-1726, 2008.

[15] D. Qian and X. Li, "Periodic solutions for ordinary differential equations with sublinear impulsive effects," Journal of Mathematical Analysis and Applications, vol. 303, no. 1, pp. 288-303, 2005.

[16] J. Yan, A. Zhao, and J. J. Nieto, "Existence and global attractivity of positive periodic solution of periodic single-species impulsive Lotka-Volterra systems," Mathematical and Computer Modelling, vol. 40, no. 5-6, pp. 509-518, 2004.

[17] S. Mohamad, K. Gopalsamy, and H. Akça, "Exponential stability of artificial neural networks with distributed delays and large impulses," Nonlinear Analysis: Real World Applications, vol. 9, no. 3, pp. 872-888, 2008.

[18] J. J. Nieto and R. Rodríguez-López, "Boundary value problems for a class of impulsive functional equations," Computers \& Mathematics with Applications, vol. 55, no. 12, pp. 2715-2731, 2008.

[19] J. Li, J. J. Nieto, and J. Shen, "Impulsive periodic boundary value problems of first-order differential equations," Journal of Mathematical Analysis and Applications, vol. 325, no. 1, pp. 226-236, 2007.

[20] A. R. Aftabizadeh, S. Aizicovici, and N. H. Pavel, "On a class of second-order anti-periodic boundary value problems," Journal of Mathematical Analysis and Applications, vol. 171, no. 2, pp. 301-320, 1992.

[21] D. Franco, J. J. Nieto, and D. O’Regan, "Anti-periodic boundary value problem for nonlinear first order ordinary differential equations," Mathematical Inequalities $\mathcal{E}$ Applications, vol. 6, no. 3, pp. 477 485, 2003.

[22] T. Jankowski, “Ordinary differential equations with nonlinear boundary conditions of antiperiodic type," Computers \& Mathematics with Applications, vol. 47, no. 8-9, pp. 1419-1428, 2004.

[23] Y. Yin, "Remarks on first order differential equations with anti-periodic boundary conditions," Nonlinear Times and Digest, vol. 2, no. 1, pp. 83-94, 1995.

[24] Y. Yin, "Monotone iterative technique and quasilinearization for some anti-periodic problems," Nonlinear World, vol. 3, no. 2, pp. 253-266, 1996.

[25] W. Ding, Y. Xing, and M. Han, "Anti-periodic boundary value problems for first order impulsive functional differential equations," Applied Mathematics and Computation, vol. 186, no. 1, pp. 45-53, 2007.

[26] B. Ahmad and J. J. Nieto, “Existence and approximation of solutions for a class of nonlinear impulsive functional differential equations with anti-periodic boundary conditions," Nonlinear Analysis: Theory, Methods \& Applications, vol. 69, no. 10, pp. 3291-3298, 2008. 
[27] D. Franco, J. J. Nieto, and D. O'Regan, “Existence of solutions for first order ordinary differential equations with nonlinear boundary conditions," Applied Mathematics and Computation, vol. 153, no. 3, pp. 793-802, 2004.

[28] Y. Chen, J. J. Nieto, and D. O'Regan, “Anti-periodic solutions for fully nonlinear first-order differential equations," Mathematical and Computer Modelling, vol. 46, no. 9-10, pp. 1183-1190, 2007.

[29] C. Ou, "Anti-periodic solutions for high-order Hopfield neural networks," Computers \& Mathematics with Applications, vol. 56, no. 7, pp. 1838-1844, 2008.

[30] R. Wu, "An anti-periodic LaSalle oscillation theorem," Applied Mathematics Letters, vol. 21, no. 9, pp. 928-933, 2008.

[31] Y. Li and L. Huang, "Anti-periodic solutions for a class of Liénard-type systems with continuously distributed delays," Nonlinear Analysis: Real World Applications. In press.

[32] K. Wang, "A new existence result for nonlinear first-order anti-periodic boundary value problems," Applied Mathematics Letters, vol. 21, no. 11, pp. 1149-1154, 2008.

[33] C. Ahn and C. Rim, "Boundary flows in general coset theories," Journal of Physics A, vol. 32, no. 13, pp. 2509-2525, 1999.

[34] A. Abdurrahman, F. Anton, and J. Bordes, "Half-string oscillator approach to string field theory (ghost sector. I)," Nuclear Physics B, vol. 397, no. 1-2, pp. 260-282, 1993.

[35] D. Franco and J. J. Nieto, "First-order impulsive ordinary differential equations with anti-periodic and nonlinear boundary conditions," Nonlinear Analysis: Theory, Methods E Applications, vol. 42, no. 2, pp. 163-173, 2000.

[36] Z. Luo, J. Shen, and J. J. Nieto, "Antiperiodic boundary value problem for first-order impulsive ordinary differential equations," Computers \& Mathematics with Applications, vol. 49, no. 2-3, pp. 253261, 2005.

[37] W. Wang and J. Shen, "Existence of solutions for anti-periodic boundary value problems," Nonlinear Analysis: Theory, Methods \& Applications, vol. 70, no. 2, pp. 598-605, 2009.

[38] N. G. Lloyd, Degree Theory, Cambridge University Press, Cambridge, UK, 1978, Cambridge Tracts in Mathematics, no. 7 